\title{
Spectral Analysis of Synthetic Power Signals of Different Regions Using Parametric Methods and Spectrogram Representations
}

\author{
Md. Shofiqul Islam ${ }^{1}$, Dr. Celia Shahnaz ${ }^{2}$, Md. Ziaul Hoque ${ }^{3}$ \\ ${ }^{[1,3]}$ (Department of Electrical and Electronic Engineering, International Islamic University Chittagong, \\ Bangladesh) \\ ${ }^{2}$ (Department of Electrical and Electronic Engineering, Bangladesh University of Engineering and Technology, \\ Bangladesh)
}

\begin{abstract}
Now a days, frequency or spectral measure is one of the most important issues of the different regions of power system. The Power spectral density (PSD) estimation can give a direct indication of major areas of different power system contributing to the oscillations in a specific mode frequency, and therefore may be used in the future for determining control actions. PSD analysis also gives information on the characteristics of power signals used in control rooms for monitoring of power system dynamics thus can aid in improving the operational reliability of interconnected power system. Therefore, in this Paper, we concentrate on the study of spectral behavior of power signals of different regions such as US Eastern Interconnection, the Nordic Power system and Nigerian power system. Among different spectral estimation methods, parametric technique has been used in this Paper to estimate PSD of power signals of different regions. Parametric methods can yield higher resolutions than nonparametric methods in cases when the signals length is short as in case of blocks of power signals. These methods use a different approach to spectral estimation; instead of trying to estimate the PSD directly from the data, they model the data as the output of a linear system driven by white noise, and then attempt to estimate the parameters of that linear system. Another approach of presenting PSD, namely, spectrogram, a time-varying spectral representation that shows how the spectral density of a signal varies with time. Detail MATLAB simulations are carried out for synthetic power signals of different regions as mentioned above to investigate the performance of parametric methods in estimating PSD and to display spectrograms thus comparing spectral behavior of different regions.
\end{abstract}

Keywords: Spectral analysis of the power system, parametric spectral estimation methods, simulation results and comparisons of the parametric method and spectrogram in different regions using synthetic power signals.

\section{Introduction}

In statistical signals processing, statistics, and physics, the spectrum of a time-series or signals is a positive real function of a frequency variable associated with a stationarystochastic process, or a deterministic function of time, which has dimensions of power per hertz $(\mathrm{Hz})$, or energy per hertz. Intuitively, the spectrum decomposes the content of a stochastic process into different frequencies present in that process, and helps identify periodicities. More specific terms which are used are the power spectrums, spectral density, power spectral density, or energy spectral density with the application of advanced signals processing techniques to power spectral density (PSD); it is possible to obtain crucial information about power system dynamic behavior. This information when incorporated in energy management system can aid in improving the operational reliability of interconnected power system, and in addition, it may also be used to characterize power system dynamics. With the initiatives carried out in the Western Electricity Coordinating Council (WECC)[1] for over two decades and others (see references [2], and [3], [4]). The application of advanced signals processing techniques has been proven successful for the estimation of mode properties, i.e. mode frequency, damping and more recently mode shape [5]. The techniques used on spectral estimation [6] are a subset of signals processing methods for linear prediction coefficient, auto regressive modeling, linear filters, and power spectral density which have a relatively long history of their own[7],[8]. The fundamental assumption is that there are random changes, mostly comprised of load variations, which excite the small-signals dynamics of a power system. Hence, spectral analysis can be used to estimate the inherent oscillatory modes of the power system [9].

\section{Headings}

Spectral estimation can be defined as estimating the distribution of total power over frequency bins of a finite-length record of a second-order stationary random process, i.e. finding the density of power in narrow spectral bands. Spectral estimation methods find application in many diverse fields such as speech processing, electromagnetics, communications, and economics, medicine, meteorology, astronomy, radar and sonar systems. Spectral processing techniques have been successfully applied to characterize the small signals oscillatory 
modes in the US WECC interconnection [1], [10] the US Eastern Interconnection [11], [12] as well as in the Australian power system [13], [14]. In this Paper, we present results of the application of parametric methods for mode frequency estimation [10] in several power systems. We emphasize the analysis of synthetic power signals with frequency originating from the U.S. Eastern Interconnection (EI), the Western Electricity Coordinating Council (WECC), the Nordic Power System, and the Nigerian power system [16]. This Paper intends to illustrate how commercially available signals processing tools are readily applicable for PSD estimation and spectrogram representations (with the pre-requisite that users are knowledgeable of signals processing methods and are familiar with the MATLAB programming language [15]) and that they produce excellent results for estimating power system modes [16]. From the available spectral analysis methods used, here we focus on the use of parametric methods, such as the Yule-Walker (YW), Burg, linear prediction, covariance, modified covariance, PRONY [17] and Steiglitz-McBride iterative methods [6], [9], [16] to find the prominent peaks in PSD plots [14],[16] and concentration of high energy in spectrogram representations. Both the spectral peaks in PSD and energy concentration in spectrogram are expected to vary in different regions of power systems. A second goal of this Paper is to share our knowledge on how to critically analyze the results from applying these techniques, and how to deal with several difficulties that may arise when analyzing different methods. The discussions and result in this Paper are of values to power system operators and planners as they provide information of the applicability of these techniques via readily available signals processing tools.

\section{Parametric Methods of PSD Estimation And Spectrogram Representation}

There are two approaches in spectral analysis: non-parametric and parametric methods. Non-parametric methods makes no assumption on data and uses basic definitions of Power Spectral Density (PSD) while parametric methods postulate some models for the data and find the parameters in the model [18].Parametric methods outperform the non-parametric methods if data satisfies the assumed model, i.e. model postulated on data is appropriate, otherwise, non-parametric methods provide better spectral estimates than parametric methods. In cases where a priori knowledge about the signals is available, it may be better to use parametric methods to obtain better spectral estimates even when it is not easy or feasible to obtain large data set [2].In this chapter, the most common terms used in spectral analysis of the power systems, parametric and nonparametric spectral analysis methods, spectrogram representations for synthetic power signals will be introduced along with their advantages and disadvantages. The energy spectral density [8], [19] describes how the energy of a signals or a time series is distributed withf $(\omega)$ frequency.The energy spectral density of the signals is the square of the magnitude of the Fourier transform of the signals [8].

$$
\hat{\mathrm{x}}(\omega)=\frac{1}{2 \pi}\left|\int_{-\infty}^{\infty} \mathrm{x}(\mathrm{t}) \mathrm{e}^{-\mathrm{jwt}} \mathrm{dt}\right|^{2}=\frac{1}{2 \pi} \hat{\mathrm{x}}(\omega) \hat{\mathrm{x}} *(\omega)
$$

Where $\omega$ is the angular frequency, (i.e., $2 \pi$ times the ordinary frequency $\hat{x}(\omega)$ is the Fourier transformofx $(\omega)$, and $\hat{\mathrm{x}} *(\omega)$ is its complex conjugate. As is always the case, the multiplicative factor of $1 / 2 \pi$ is not universally agreed upon, but rather depends on the particular normalizing constants used in the definition of the various Fourier transforms. Thedefinition of energy spectral density is most suitable for transients, i.e., pulselike signals, for which the Fourier transforms of the signals exist. For continued signals that describe, for example, stationary physical processes, it makes more sense to define a power spectral density (PSD), which describes how the power [2] of a signals or time series is distributed over the different frequencies. Fourier transform exists,

$$
F_{x}^{T}(\omega)=\frac{1}{\sqrt{T}} \int_{0}^{T} x(t) \exp (-j \omega t) d t
$$

Which is not necessarily the case; we can define the power spectral density as

$$
f(\omega)=\lim _{\mathrm{T} \rightarrow \infty} E\left[\left|\mathrm{~F}_{\mathrm{x}}^{\mathrm{T}}(\omega)\right|^{2}\right]
$$

Some properties of the PSD include: the spectrum of a real valued process is symmetricf $(-\omega)=f(\omega)$, or in other words, it is an even function, if the process is continuous and purely in deterministic, the auto covariance function can be reconstructed by using the Inverse Fourier transform, It describes the distribution of the varianceover frequency. In particular [8],

$$
\operatorname{Var}\left(\mathrm{X}_{\mathrm{n}}\right)=\gamma_{0}=2 \int_{0}^{1 / 2} \mathrm{f}(\omega) \mathrm{d} \omega
$$

$\gamma(\mathrm{t})=\alpha_{1} \gamma_{1}(\tau)+\alpha_{2} \gamma_{2}(\tau) \mathrm{f}=\alpha_{1} \mathrm{f}_{1}+\alpha_{2} \mathrm{f}_{2}$, it is a linear function of the auto covariance function, then If $\gamma$ is decomposed into two functions, thenWheref $f_{i}=\hat{\gamma}_{\mathrm{i}}$ PSD estimation. The goal of power spectral density estimation is to estimate the spectral density of a random signal from a sequence of time samples. Depending on what is known about the signals, estimation techniques can involve parametric or non-parametric approaches [9], and may be based on time-domain or frequency-domain analysis. For example, a common parametric technique involves fitting the observations to an autoregressive model.Parametric methods can yield higher resolutions than nonparametric methods [9] in cases when the signals length is short. These methods use a different approach to spectral estimation [8]; instead of trying to estimate the PSD directly from the data, they model the 
data as the output of a linear system driven by white noise, and then attempt to estimate the parameters of that linear system [20].The most commonly used linear system model is the all-pole model, a filter with all of its zeroes at the origin in the z-plane. The output of such a filter for white noise input is an autoregressive (AR) process. For this reason, these methods are sometimes referred to as AR methods of spectral estimation. The AR methods tend to adequately describe spectra of data that is "peaky," that is, data whose PSD is large at certain frequencies [15]. The data in many practical applications (such as speech) tends to have "peaky spectra" so that AR models are often useful. In addition, the AR models lead to a system of linear equations which is relatively simple to solve [9], [15]. Signals Processing Toolbox AR methods for spectral estimation include: Covariance method, Modified covariance method, Yule-Walker AR method (autocorrelation method), Burg method [9],[15]. All AR methods yield a PSD estimate given by

$$
\widehat{P}(f)=\frac{1}{F_{s}} \frac{p}{\left|1-\sum_{k=1}^{p} \hat{a}_{p}(k) e^{-j 2 \pi k f / F s}\right|^{2}}
$$

The different AR methods estimate the parameters slightly differently, yielding different PSD estimates. The following table provides a summary of the different AR methods [15]. The PSD estimation of power signals can be incorporated in energy measurement systems thus can aid in improving the operational reliability of interconnected power system. It may also be used to characterize power system dynamics. PSD estimation can give a direct indication of major areas of different power system contributing to the oscillations in a specific mode frequency, and therefore may be used in the future for determining control actions. Estimation of mode frequencies and damping helps to analysis of power measurement in different power systems [5]. Among different methods, parametric technique has been used to estimate power spectral density in different power systems. In the literature (see reference [1],[3]), the PSD estimation techniques has been applied successfully to estimate different mode properties of different power systems, such as AR coefficient, mode frequency, damping and mode shape [5], [8],[15] .AR modeling is used in parametric methods for PSD estimation of power signals. An autoregressive (AR) model is a type of random process which is often used to model and predict various types of natural phenomena. The autoregressive model uses linear prediction formulas that attempt to predict an output of a system based on the previous outputs. Power signals can be model as an output of autoregressive model.The notation AR (p) indicates an autoregressive model of order $\mathrm{p}$. The AR (p) model is defined as

$$
\mathrm{X}_{\mathrm{t}}=\mathrm{c}+\sum_{\mathrm{i}=1}^{\mathrm{p}} \varphi_{\mathrm{i}} \mathrm{X}_{\mathrm{t}-\mathrm{i}}+\varepsilon_{\mathrm{t}}
$$

Where $\varphi_{1, \cdots,}, \varphi$ the parameters of the model are, $\mathrm{c}$ is a constant (often omitted for simplicity) and $\varepsilon_{\mathrm{t}}$ is white noise. An autoregressive model can thus be viewed as the output of an all-poleinfinite impulse response filter whose input is white noise [22].Characteristic polynomial: The autocorrelation function of an AR (p) process can be expressed as

$$
\rho(\tau)=\sum_{\mathrm{k}=1}^{\mathrm{p}} \mathrm{a}_{\mathrm{k}} \mathrm{y}_{\mathrm{k}}-|\tau|
$$

Where $y_{k}$ are the roots of the polynomial,

$$
\emptyset(\mathrm{B})=1-\sum_{\mathrm{k}=1}^{\mathrm{p}} \varphi_{\mathrm{k}} \mathrm{B}^{\mathrm{k}}
$$

A spectrogram is a time-varying spectral representation [9] (forming an image) that shows how the spectral density of a signal varies with time. Also known as spectral waterfalls, sonograms, voiceprints, or voicegrams, spectrograms are used to identify phonetic sounds, to analyze the cries of animals; they were also used in many other fields including music, sonar/radar, speech processing,[21] seismology, etc. The instrument that generates a spectrogram is called a spectrograph [8].The spectrogram of a signal $s(t)$ can be estimated by computing the squared magnitude of the STFT of the signalss $(t)$, as follows: [15]

$$
\operatorname{spectrogram}(t, \omega)=|\operatorname{STFT}(t, \omega)|^{2}
$$

\section{Results And Performance Comparisons}

We discuss PSD estimates of synthetic power signals of three different regions, namely US Eastern Interconnection, Nordic system and Nigerian system. US Eastern Interconnection System: It is observed that the frequency regulation band in the US Eastern Interconnection ranged from 59.95 to $60.05 \mathrm{~Hz}(\Delta \mathrm{f}=0.1 \mathrm{~Hz})$, Nordic System: While the frequency regulation band in the Nordic system varied from 49.9 to $50.15 \mathrm{~Hz}(\Delta \mathrm{f}=$ $0.25 \mathrm{HzNigeria}$ System: Compared with these power systems, the frequency in Nigeria is loosely regulated with variations ranging from 49 to $51 \mathrm{~Hz}$, a $1 \mathrm{~Hz}$ variation from steady state of $50 \mathrm{~Hz}$. In addition to the power to upper and lower bounds of frequency variation, it is also important to note that frequency can vary between 0.4$0.5 \mathrm{~Hz}$ in $1 \mathrm{~min}$. window. In contrast, both the US EI and the Nordic system have a tightly regulated operation maintaining frequency variations closely between the frequencies bands discussed. Now In this chapter, we like to see the frequency change of difference regions by PSD estimation [3]. 


\section{A. Results using covariance method}

In Fig: 01, the PSD estimation of synthetic power signals obtained using covariance method for US EI is shown. Then we find out the desirable peak from PSD plot obtained using the Covariance Method.

\section{For US Eastern Interconnection}

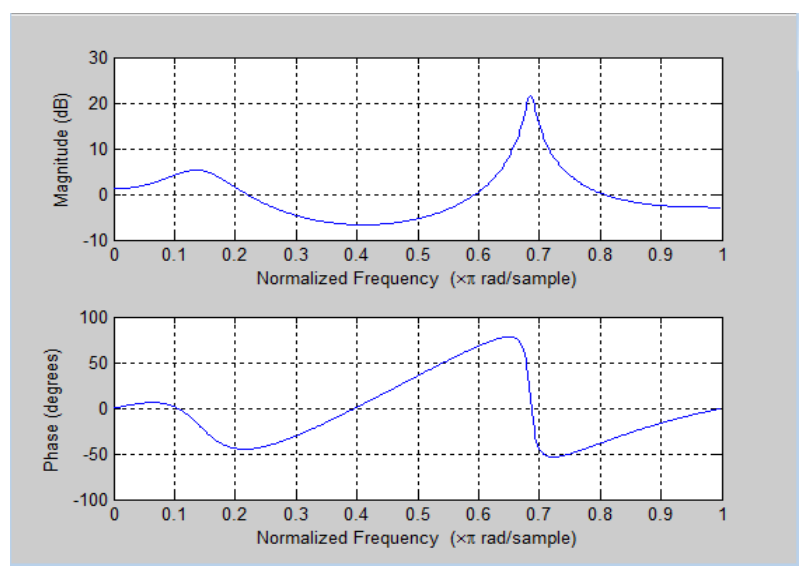

Fig 01: PSD estimation from AR coefficients obtained using covariance method for US EI.

In Fig 03, the PSD estimation of synthetic power signals obtained using covariance method for Nigerian system is shown. Then we find out the desirable peak from PSD plot obtained using the Covariance Method

\section{For Nigerian System}

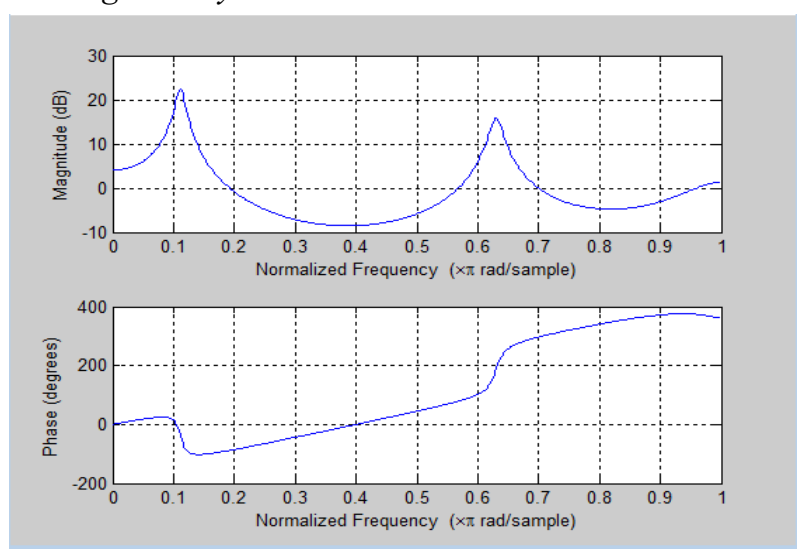

Fig 03: PSD from AR coefficients obtained using Covariance method for Nordic.
In Fig 02, the PSD estimation of synthetic power signals obtained using covariance method for Nordic system is shown. Then we find out the desirable peak from PSD plot obtained using the Covariance Method.

\section{For Nordic System}

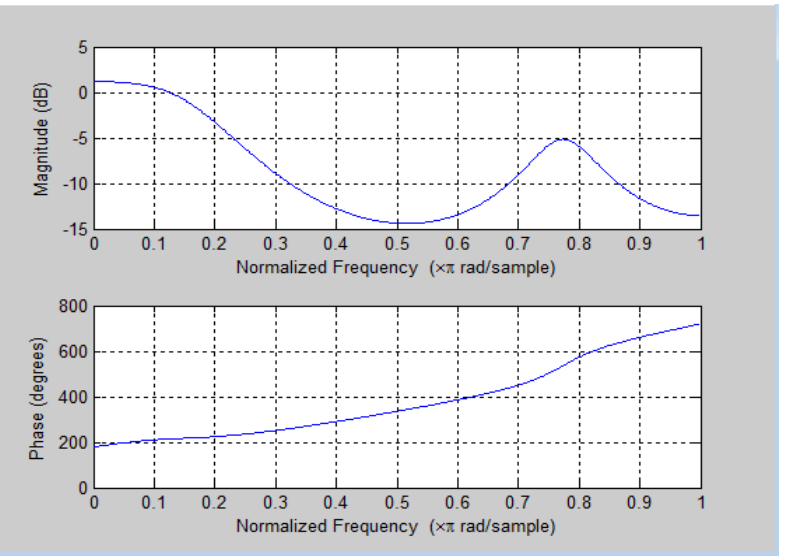

Fig 02: PSD from AR coefficients obtained using Covariance method for Nordic.

In Table 01, PSD peak positions of different regions obtained using the Covariance method are summarized.

Table 01: Comparisons of PSD peaks obtained using Covariance method for different regions.

\begin{tabular}{|l|l|l|}
\hline Region & \multicolumn{1}{|c|}{$\omega \mathrm{d}$} & Remark \\
\hline US EI system & $0.14,0.68$ & 2 peaks \\
\hline Nordic system & $0.11,0.78$ & 2 peaks \\
\hline Nigerian system & $0.12,0.63$ & 2 peaks \\
\hline
\end{tabular}

In table 01, by PSD estimation of synthetic power signals using Covariance method, same number of peaks (2)are obtained for different regions but at closely different positions. According to synthetic power signals, our desirable number of peaks is three. But simulation results show 2 peaks for all three regions, namely US EI system, Nordic \& Nigerian System. 


\section{B. Results using Modified Covariance method}

In Fig: 04, the PSD estimation of synthetic power signals obtained using modified covariance method for US EI is shown. Then we find out the desirable peak from PSD plot obtained using the modified covariance Method.

\section{For US Eastern Interconnection}

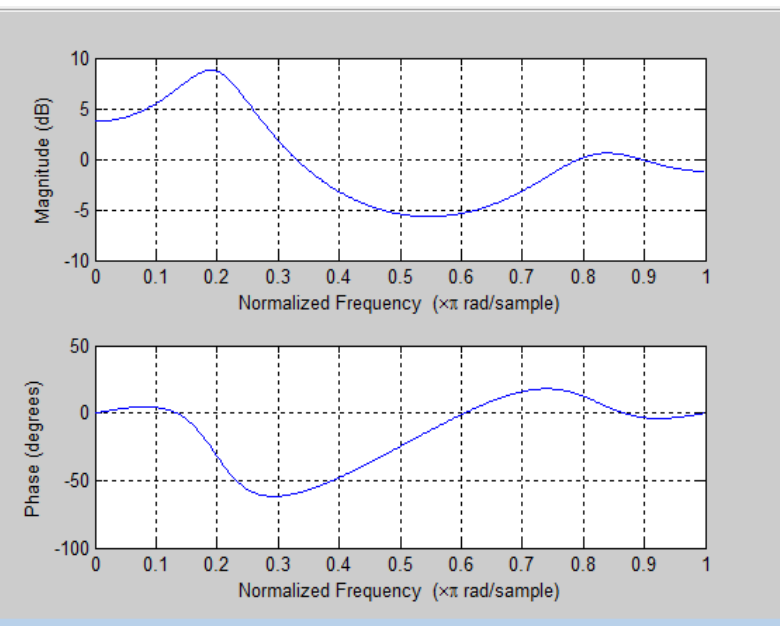

Fig 04: PSD estimation from AR coefficients obtained using modified covariance method for US EI.

In Fig 06, the PSD estimation of synthetic power signals obtained using modified covariance method for Nigerian system is shown. Then we find out the desirable peak from PSD plot obtained using the modified covarianceMethod.

\section{For Nigerian System}

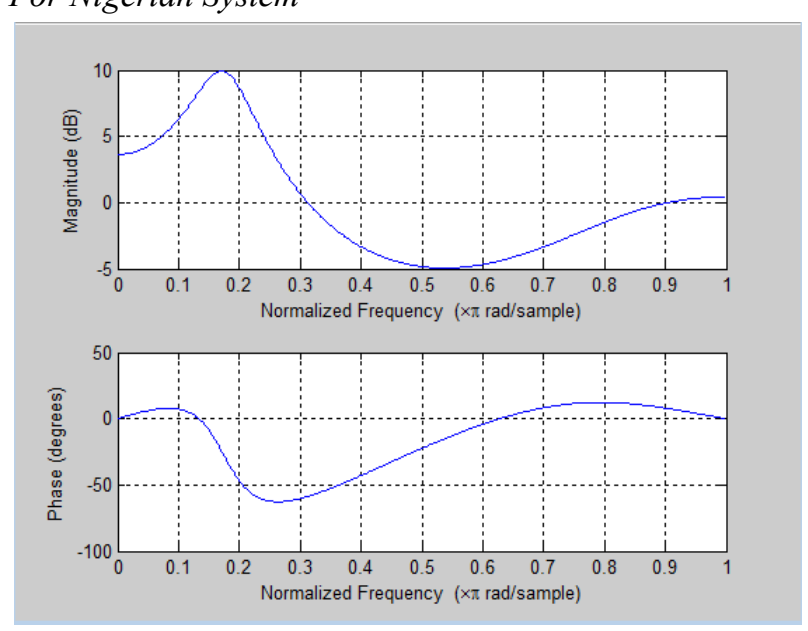

Fig 06: PSD from AR coefficients obtained using modified covariance method for Nordic.
In Fig 05, the PSD estimation of synthetic power signals obtained using modified covariance method for Nordic system is shown. Then we find out the desirable peak from PSD plot obtained using the modified covariance Method.

\section{For Nordic System}

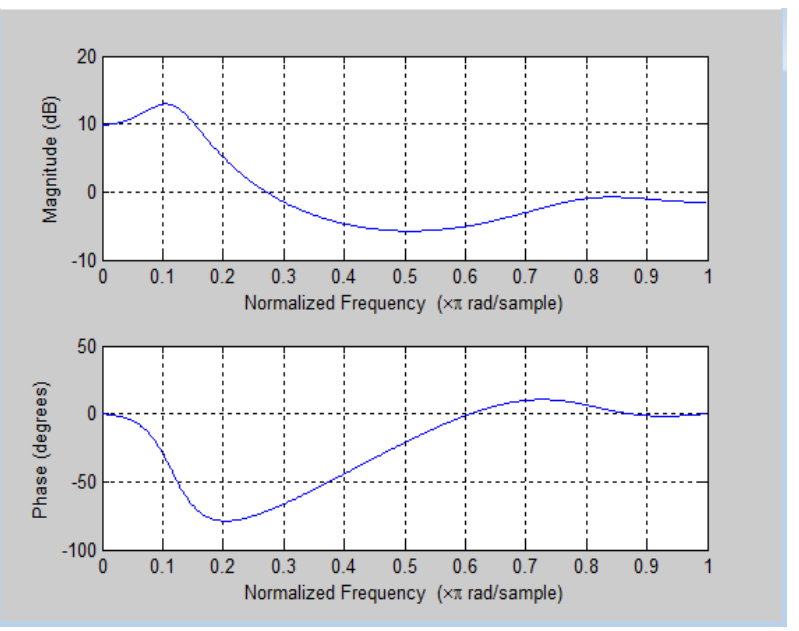

Fig 05: PSD from AR coefficients obtained using modified covariancemethod for Nordic.

In Table 02, PSD peak positions of different regions obtained using the modified covariance method are summarized.

Table 02: Comparisons of PSD peaks obtained using modified covariance method for different regions.

\begin{tabular}{|l|l|l|}
\hline Region & \multicolumn{1}{|c|}{$\omega \mathrm{d}$} & Remark \\
\hline US EI system & $0.19,0.83$ & 2 peaks \\
\hline Nordic system & $0.12,0.82$ & 12 peaks \\
\hline Nigerian system & $0.11,0.94$ & 2 peaks \\
\hline
\end{tabular}

In Table 02, by PSD estimation of synthetic power signals, using modified covariance method, same number of peaks (2) obtained for different regions but at closely different positions. According to synthetic power signals, our desirable number of peaks is three. But simulation results show 2 peaks for all three regions, namely US EI system, Nordic \& Nigerian System. 


\section{Results using Yule-Walker (YW) method}

In Fig: 07, the PSD estimation of synthetic power signals obtained using YW method for US EI is shown. Then we find out the desirable peak from PSD plot obtained using the YW Method.

\section{For US Eastern Interconnection}

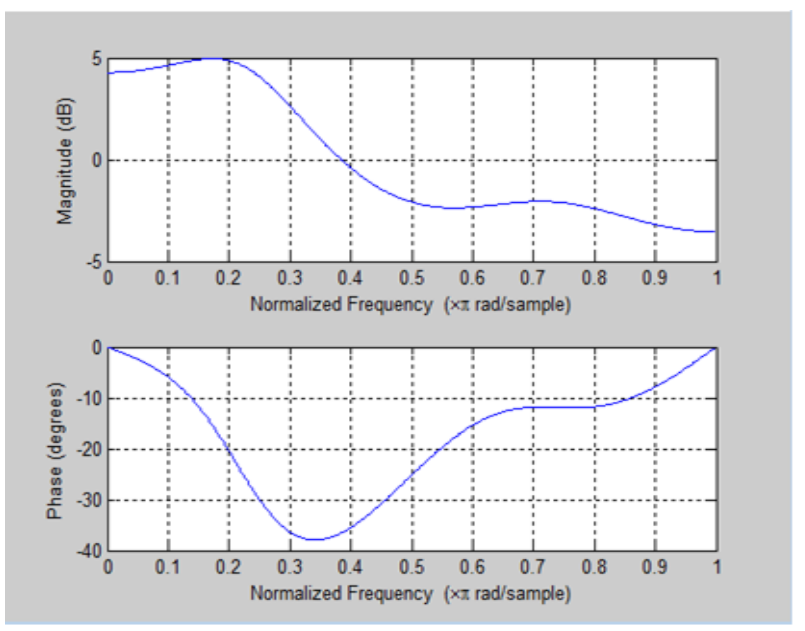

Fig 07: PSD estimation from AR coefficients obtained using YW method for US EI.

In Fig 09, the PSD estimation of synthetic power signals obtained using YW method for Nigerian system is shown. Then we find out the desirable peak from PSD plot obtained using the YW Method.

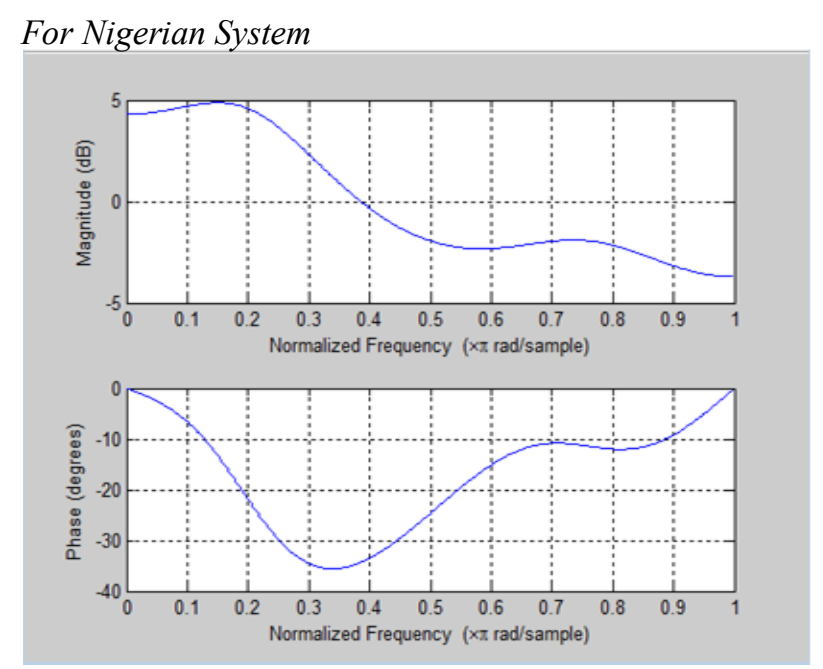

Fig 09: PSD from AR coefficients obtained using YW method for Nordic.
In Fig 08, the PSD estimation of synthetic power signals obtained using YW method for Nordic system is shown. Then we find out the desirable peak from PSD plot obtained using the YW Method.

For Nordic System

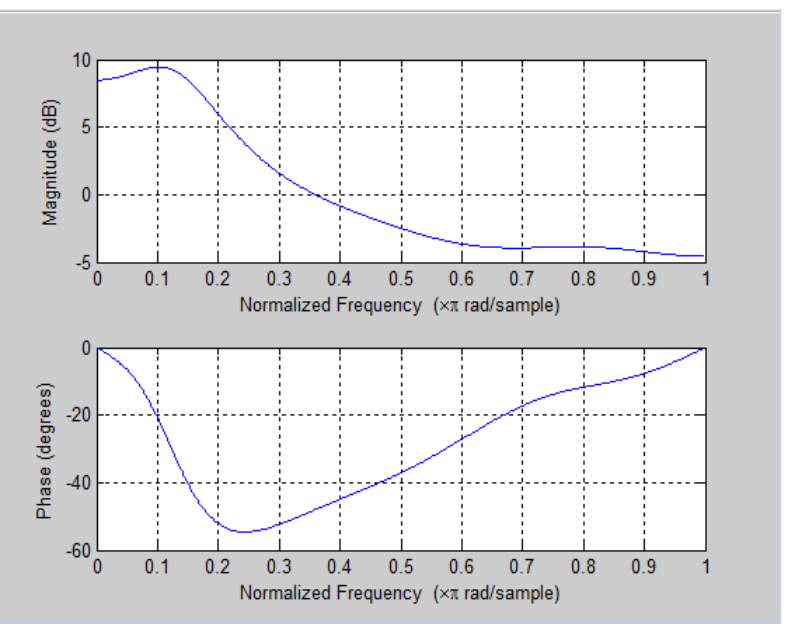

Fig 08: PSD from AR coefficients obtained using YW method for Nordic.

In Table 03, PSD peak positions of different regions obtained using the YW method are summarized.

Table 03: Comparisons of PSD peaks obtained using YW method for different regions.

\begin{tabular}{|l|l|l|}
\hline Region & \multicolumn{1}{|c|}{$\omega \mathrm{d}$} & Remark \\
\hline US EI system & $0.19,0.72$ & 2 peaks \\
\hline Nordic system & 0.12 & 1 peaks \\
\hline Nigerian system & $0.16,0.74$ & 2 peaks \\
\hline
\end{tabular}

In table 03, by PSD estimation of synthetic power signals using YW method, two peaks (2) are obtained for US EI \& Nigerian regions but at closely different positions. One peak (1) is obtained from PSD of Nordic system. According to synthetic power signals, our desirable number of peaks is three. 


\section{Results using Burg method}

In Fig: 10, the PSD estimation of synthetic power signals obtained using Burg method for US EI is shown. Then we find out the desirable peak from PSD plot obtained using the Burg Method.

\section{For US Eastern Interconnection}

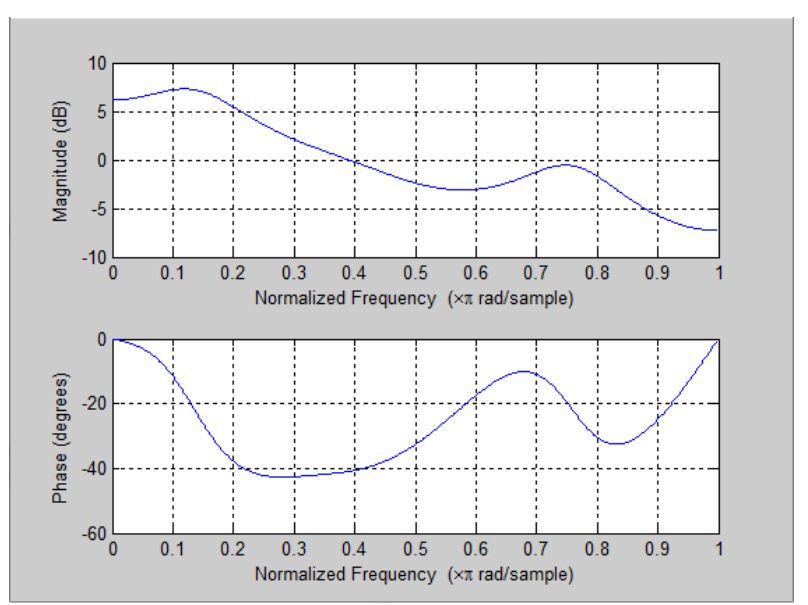

Fig 10: PSD estimation from AR coefficients obtained using Burg method for US EI.

In Fig 12, the PSD estimation of synthetic power signals obtained using Burg method for Nigerian system is shown. Then we find out the desirable peak from PSD plot obtained using the Burg Method.

\section{For Nigerian System}
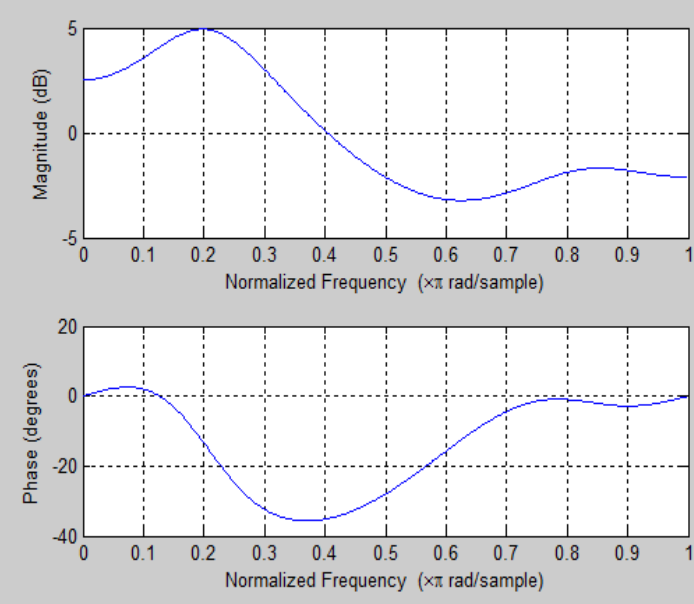

Fig 12: PSD from AR coefficients obtained using Burg method for Nordic.
In Fig 11, the PSD estimation of synthetic power signals obtained using Burg method for Nordic system is shown. Then we find out the desirable peak from PSD plot obtained using the Burg Method.

\section{For Nordic System}

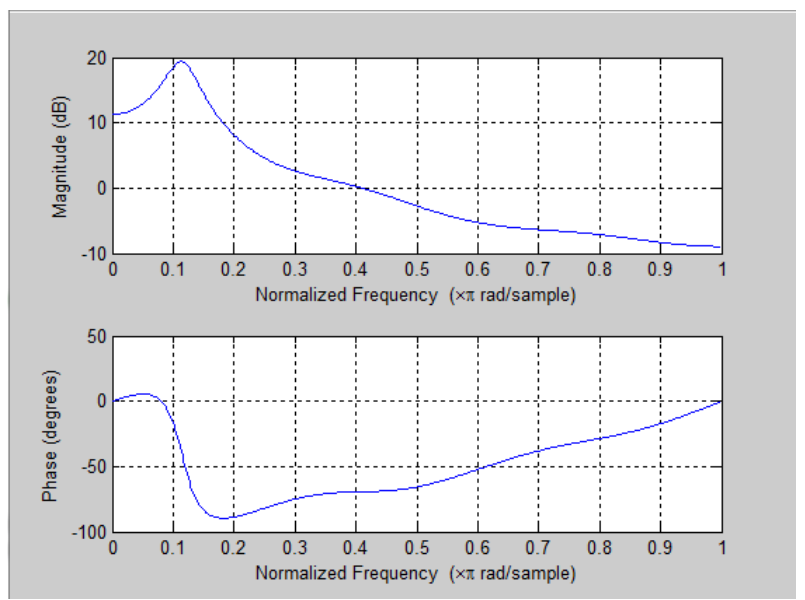

Fig 11: PSD from AR coefficients obtained using Burg method for Nordic

In Table 04, PSD peak positions of different regions obtained using the Burg method are summarized.

Table 04: Comparisons of PSD peaks obtained using Burg method for different regions.

\begin{tabular}{|l|l|l|}
\hline Region & \multicolumn{1}{|c|}{$\omega \mathrm{d}$} & Remark \\
\hline US EI system & $0.13,0.75$ & 2 peaks \\
\hline Nordic system & 0.12 & 1 peaks \\
\hline Nigerian system & $0.2,0.85$ & 2 peaks \\
\hline
\end{tabular}

According to synthetic power signals, our desirable number of peaks is three. In table 04, by PSD estimation of synthetic power signals using Burg method, two peaks (2) are obtained for US EI \& Nigerian regions but at closely different positions. One peak (1) is obtained from PSD of Nordic system. 


\section{E. Results using Linear Prediction Coefficient (LPC) method}

In Fig: 13, the PSD estimation of synthetic power signals obtained using LPC method for US EI is shown. Then we find out the desirable peak from PSD plot obtained using the LPC Method.

\section{US Eastern Interconnection}

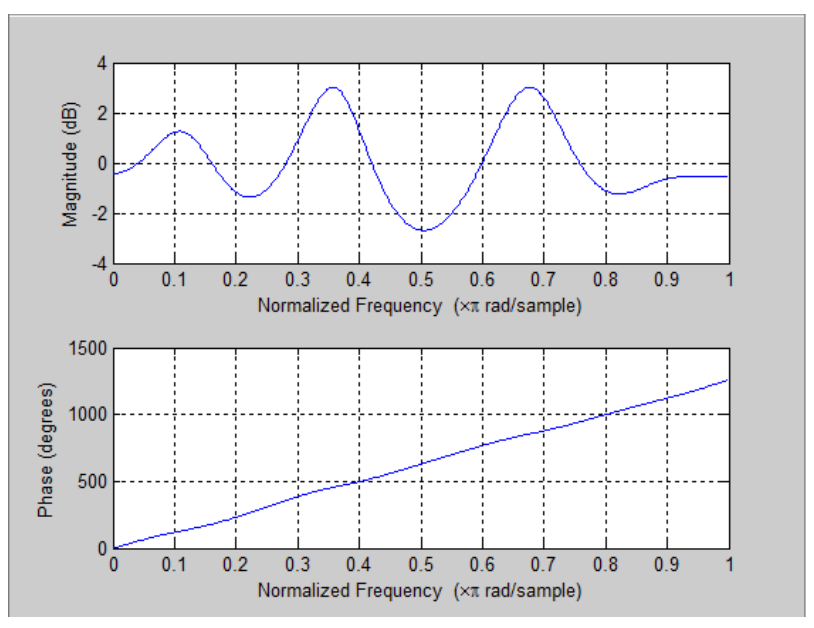

Fig 13: PSD estimation from AR coefficients obtained using LPC method for US EI.

In Fig 15, the PSD estimation of synthetic power signals obtained using LPC method for Nigerian system is shown. Then we find out the desirable peak from PSD plot obtained using the LPC Method

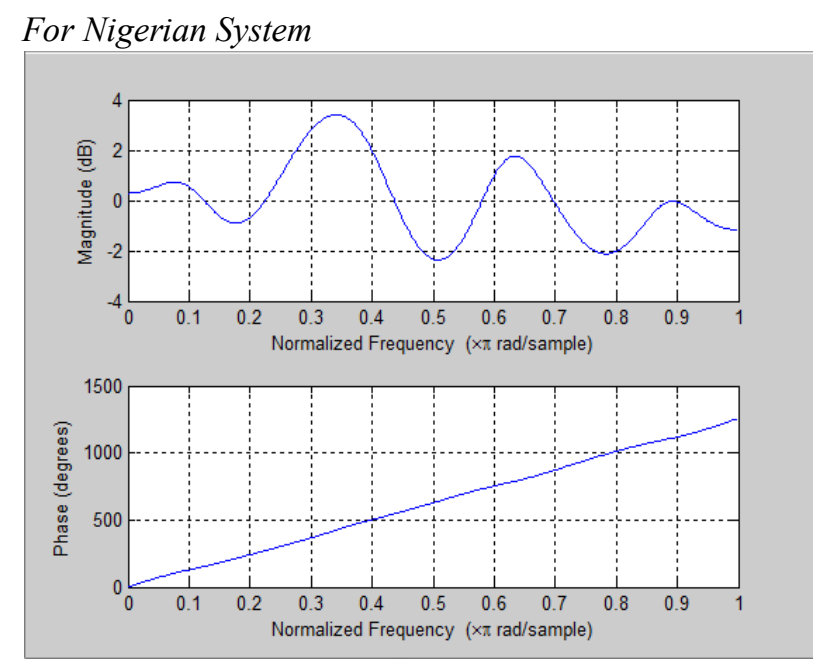

Fig 15: PSD from AR coefficients obtained using LPC method for Nordic.
In Fig 14, the PSD estimation of synthetic power signals obtained using LPC method for Nordic system is shown. Then we find out the desirable peak from PSD plot obtained using the LPC Method.

For Nordic System

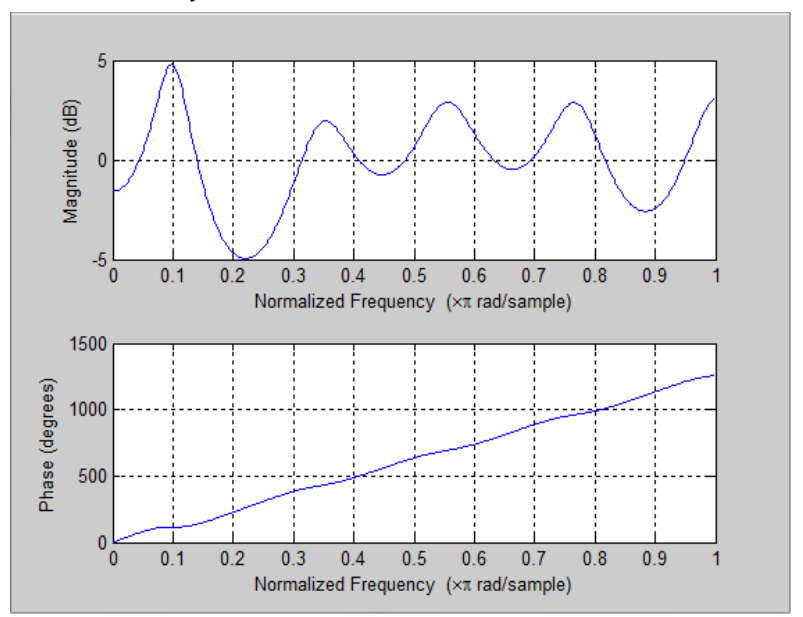

Fig 14: PSD from AR coefficients obtained using LPC method for Nordic.

In Table 05, PSD peak positions of different regions obtained using the LPC method are summarized.

Table 05: Comparisons of PSD peaks obtained using linear prediction coefficient method for different regions.

\begin{tabular}{|l|l|l|}
\hline Region & \multicolumn{1}{|c|}{$\omega \mathrm{d}$} & Remark \\
\hline US EI system & $0.12,036,0.68$ & 3 peaks \\
\hline Nordic system & $0.1,0.35,0.56,0.78$ & 4 peaks \\
\hline Nigerian system & $0.08,0.34,0.63,0.89$ & 4 peaks \\
\hline
\end{tabular}

According to synthetic power signals, our desirable number of peaks is three. In table 05, by PSD estimation of synthetic power signals using LPC method, four peaks (4) are obtained for Nordic \& Nigerian regions but at closely different positions. Three peaks (3) are obtained from PSD of US EI system. 


\section{F. Results using PRONY method}

In Fig: 16, the PSD estimation of synthetic power signals obtained using Prony method for US EI is shown. Then we find out the desirable peak from PSD plot obtained using the Prony Method.

\section{For US Eastern Interconnection}

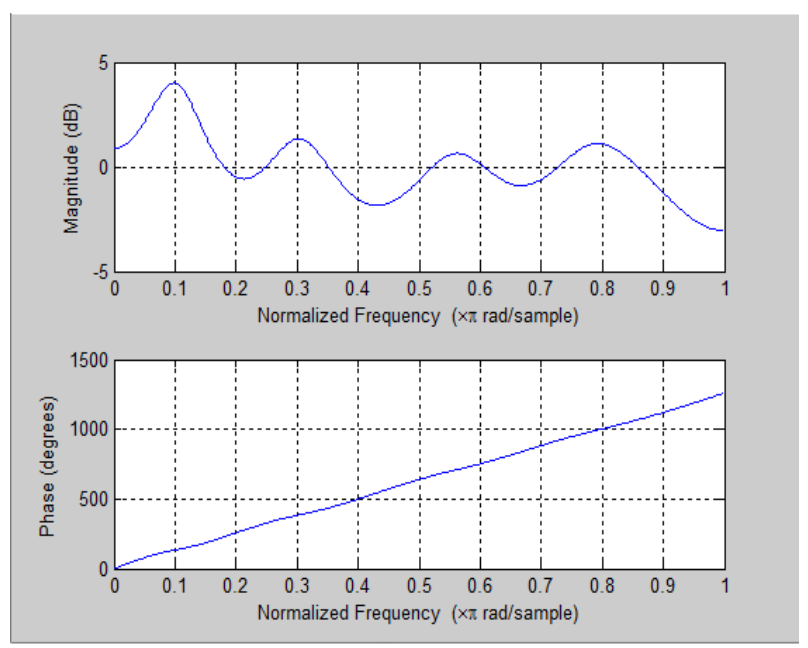

Fig 16: PSD estimation from AR coefficients obtained using Prony method for US EI.

In Fig 18, the PSD estimation of synthetic power signals obtained using Prony method for Nigerian system is shown. Then we find out the desirable peak from PSD plot obtained using the Prony Method

\section{For Nigerian System}

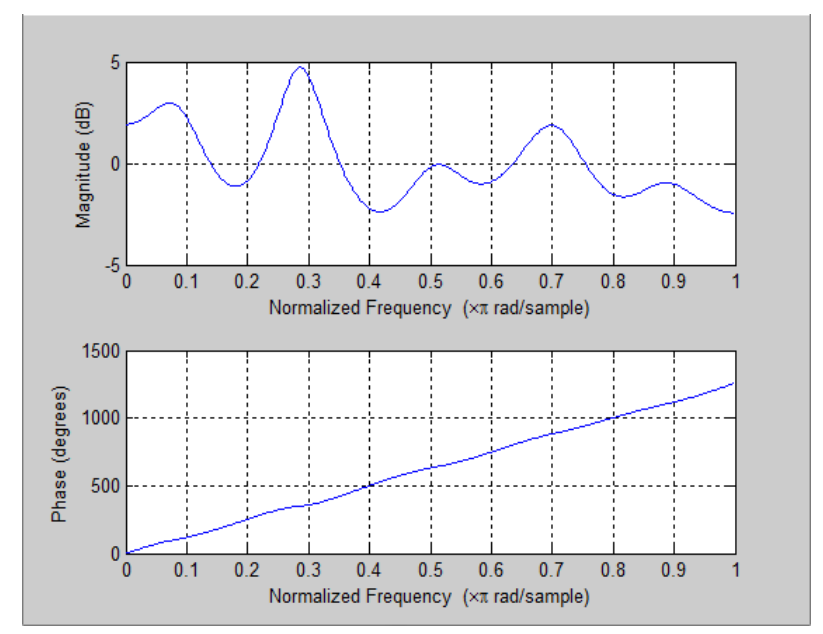

Fig 18: PSD from AR coefficients obtained using Prony method for Nordic
In Fig 17, the PSD estimation of synthetic power signals obtained using Prony method for Nordic system is shown. Then we find out the desirable peak from PSD plot obtained using the Prony Method.

\section{For Nordic System}

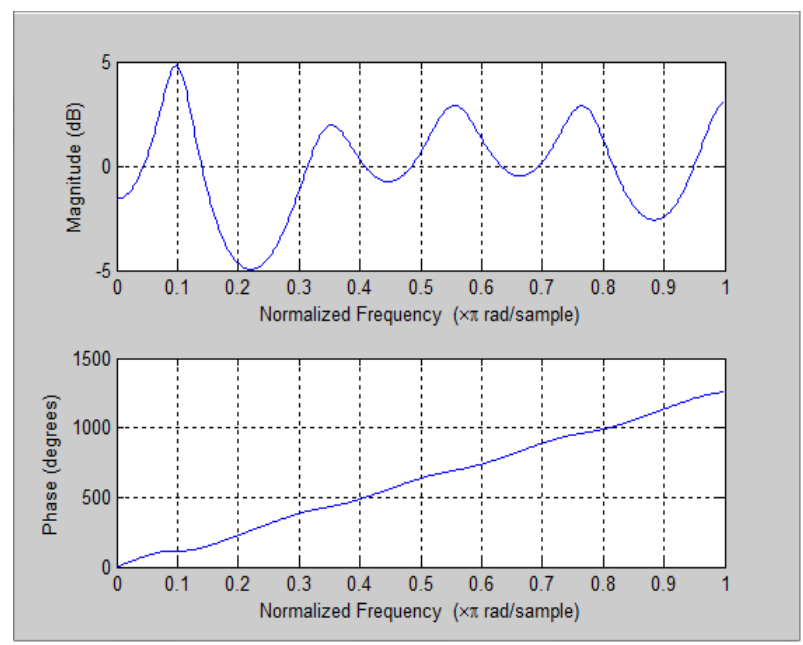

Fig 17: PSD from AR coefficients obtained using Prony method for Nordic

In Table 06, PSD peak positions of different regions obtained using the Prony method are summarized.

Table 06: Comparisons of PSD peaks obtained using PRONY method for different regions

\begin{tabular}{|l|l|l|}
\hline Region & \multicolumn{1}{|c|}{$\omega \mathrm{d}$} & Remark \\
\hline US EI system & $0.1,0.3,0.56,0.79$ & 4 peaks \\
\hline Nordic system & $0.1,0.34,0.55,0.77$ & 4 peaks \\
\hline Nigerian system & $0.08,0.28,0.51,0.7,0.89$ & 5 peaks \\
\hline
\end{tabular}

According to synthetic power signals, our desirable number of peaks is three. In table 06, by PSD estimation of synthetic power signals using PRONY method, four peaks (4) are obtained for US EI \& Nordic regions but at closely different positions. Five peaks (5) are obtained from PSD of Nigerian system. 


\section{G. Results using Steiglitz-McBride iterative (STMCB) method}

In Fig: 19, the PSD estimation of synthetic power signals obtained using STMCB method for US EI is shown. Then we find out the desirable peak from PSD plot obtained using the STMCB Method.

\section{For US Eastern Interconnection}

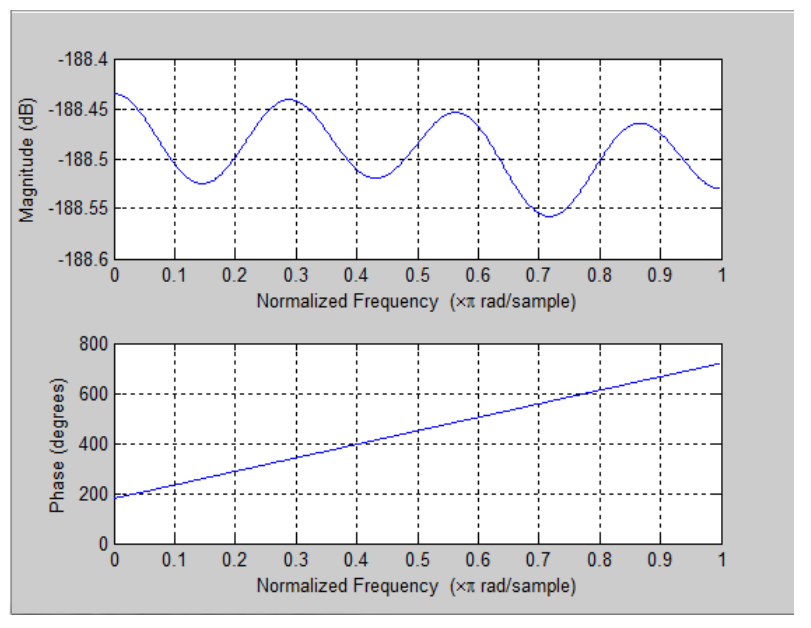

Fig 19: PSD estimation from AR coefficients obtained using STMCB method for US EI.

In Fig 21, the PSD estimation of synthetic power signals obtained using STMCB method for Nigerian system is shown. Then we find out the desirable peak from PSD plot obtained using the STMCB Method.

\section{For Nigerian System}

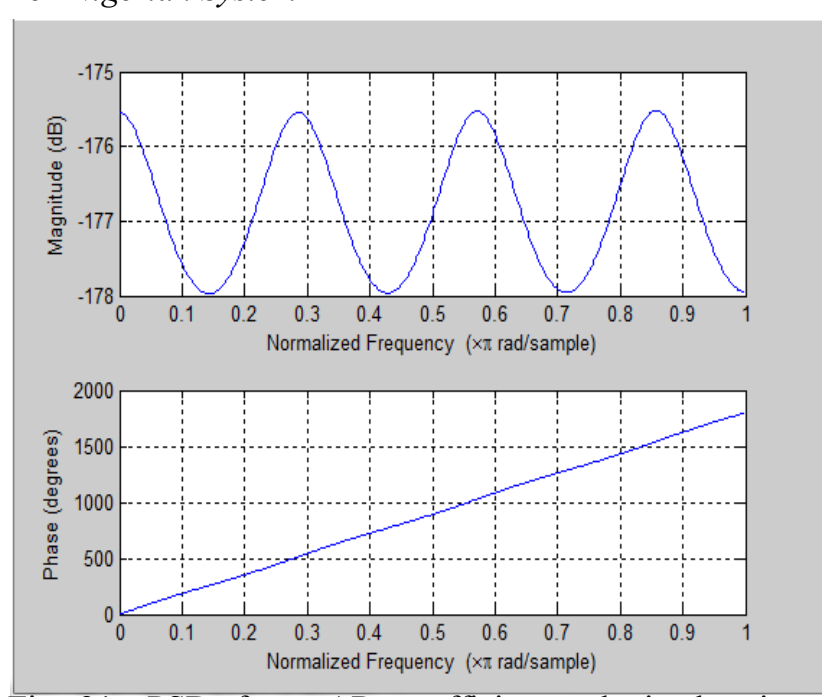

Fig 21: PSD from AR coefficients obtained using STMCB method for Nordic

\section{H. Spectrogram Representation}

In the spectrogram, the red colors represent high energy and the blue colors represent low energy of the PSD plot of the synthetic power signals.
In Fig 20, the PSD estimation of synthetic power signals obtained using STMCB method for Nordic system is shown. Then we find out the desirable peak from PSD plot obtained using the STMCB Method.

\section{For Nordic System}

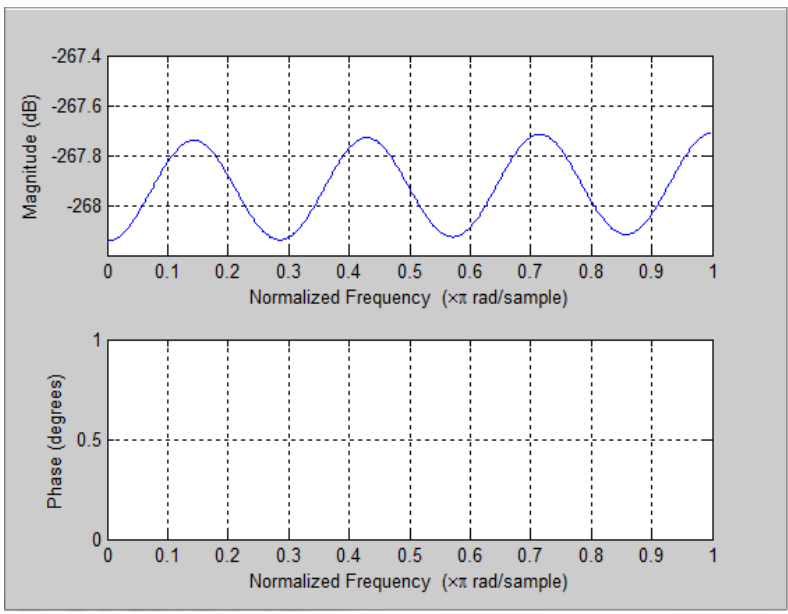

Fig 20: PSD from AR coefficients obtained using STMCB method for Nordic

In Table 07, PSD peak positions of different regions obtained using the STMCB method are summarized.

Table 07: Comparisons of PSD peaks obtained using Steiglitz-McBride iterative method for different regions.

\begin{tabular}{|l|c|l|}
\hline Region & \multicolumn{1}{|c|}{$\omega \mathrm{d}$} & Remark \\
\hline US EI system & $0.28,0.56,0.87$ & 3 peaks \\
\hline Nordic system & $0.15,0.43,0.71$ & 3 peaks \\
\hline Nigerian system & $0.28,0.57,0.85$ & 3 peaks \\
\hline
\end{tabular}

In table 07 , by PSD estimation of synthetic power signals using STMCB method, same number of peaks (2)are obtained for different regions but at closely different positions. According to synthetic power signals, our desirable number of peaks is three. But simulation results show 2 peaks for all three regions, namely US EI system, Nordic \& Nigerian System. 
In Fig. (a), spectrogram representations of synthetic power signals of US Eastern Interconnection system have been displayed.

\section{US Eastern Interconnection System}

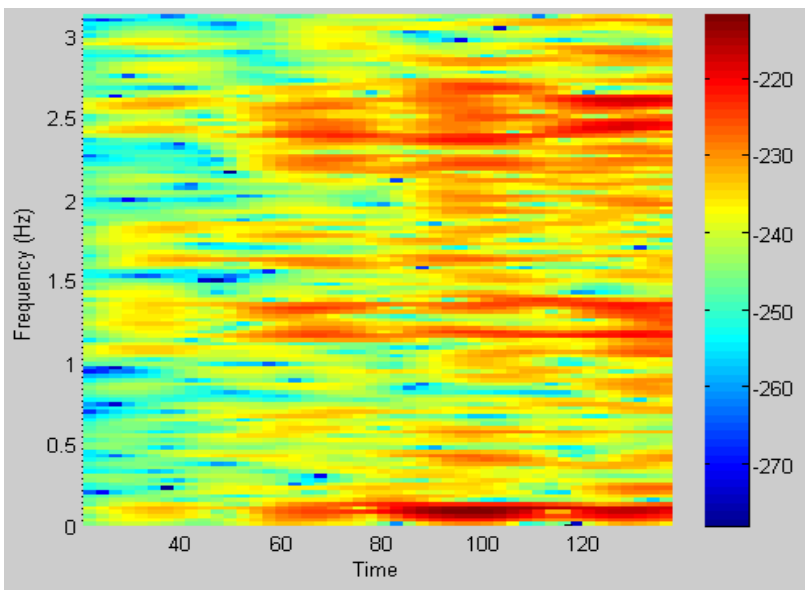

Fig. a: US Eastern Interconnection frequency range
In Fig. (b), spectrogram representations of synthetic power signals of Nordic system have been displayed.

\section{Nordic System}

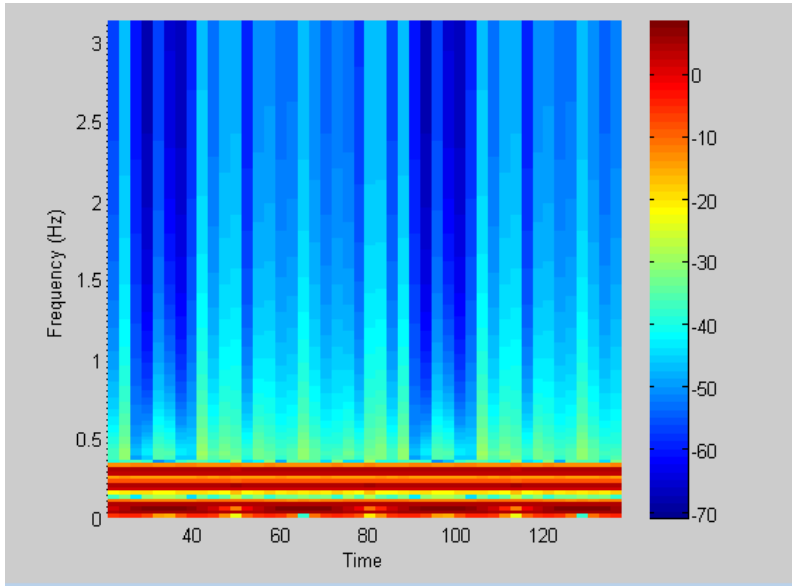

Fig. b: Nordic frequency range

Comments: High energy appears in small bands around Comments: High energy appears only in small two $0.07 \mathrm{~Hz}, 1.15 \mathrm{~Hz}, 1.35 \mathrm{~Hz}, 2.38 \mathrm{~Hz}, 2.43 \mathrm{~Hz}$, and $2.6 \mathrm{~Hz}$.

InFig. (c), spectrogram representations of synthetic power signals of Nigerian system have been displayed.

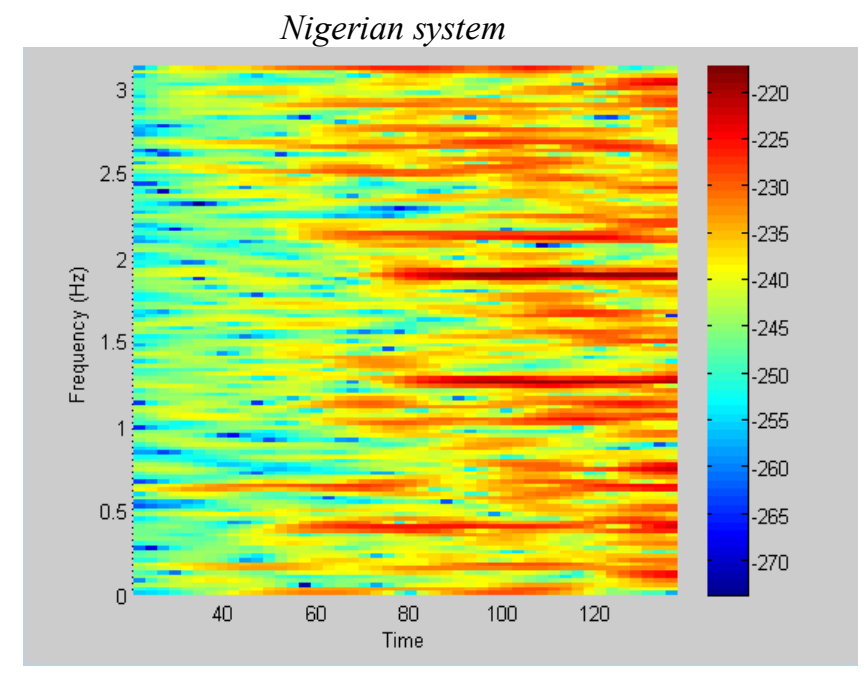

Fig. c: Nigerian frequency range

Comments: High energy appears in small bands around $0.12 \mathrm{~Hz}, 0.39 \mathrm{~Hz}, 0.76 \mathrm{~Hz}, 1.276 \mathrm{~Hz}, 1.914 \mathrm{~Hz}, 2.11 \mathrm{~Hz}$, $2.651 \mathrm{~Hz}$, and $3.019 \mathrm{~Hz}$.

\section{Conclusion}

In this Paper, spectral analysis of power systems of different regions is performed using parametric methods and spectrogram representations. Simulation results show that Steiglitz-McBride iterative method is the best PSD estimation than other parametric methods. Spectrogram representations of power signals help out to understand high or low energy band of different regions. Thus the analysis and discussion in this Paper provided an insight on how to critically analyze the results derived from different PSD estimation techniques and spectrogram plots. However, the presence of sinusoids in real power data will ultimately pose a challenge for PSD estimation and spectrogram analysis thus opening a scope for further research 


\section{Acknowledgements}

All praises and pride are for the almighty Allah, who enabled us blessing, dedication and motivation to fulfill our goals in completing the paper. We would like to express our sincere gratitude and profound indebtedness to our supervisor Dr. Celia Shahnaz, Associate professor of the Dept. of EEE, Bangladesh University of Engineering \& Technology (BUET) for her guidance, encouragement, constructive suggestion, and support during the span of this research. Among many things we learned from Dr. Celia Shahnaz, persistent effort is undoubtedly the most important one, which enables us to sort out several important issues in the area of signal processing. We believe our work in the past one year advanced the state of art of the signal process problem. We also want to thank her for spending so many hours with us in exploring new areas of research and new ideas and improving the writhing of this dissertation. We would also like to thanks our honorable head of department Muhammad AtharUddin and who helped us in our paper as well as sharing their expertise and their knowledge of the subject which allowed us to complete our paper effectively in time. We also want to thank Dr. ShaikhAnowarul Fattah, Dept. of EEE, BUET. And we are also very thankful to Professor, Dr. AminulHoque, Dept. of EEE, and BUET for his effective advice. Most importantly, we wish to thank our parents, for being our driving force and standing by us through thick and thin. Without them we would never have come so far in pursuing my dream.

\section{References:}

[1] L. Dosiek, D. Trudnowski, and J. Pierre, "New algorithms for mode shape estimation using measured data," in IEEE Power and Energy Society General Meeting, July 2008, pp. 1-8.

[2] D. Trudnowski and J.W. Pierre, in Inter-area Oscillations in Power Systems: A Nonlinear and Nonstationary Perspective, ser. Power Electronics and Power Systems, A. R. Messina, Ed. Springer, 2009, ch. Signal Processing Methods for Estimating Small-Signal Dynamic Properties from Measured Responses, pp. 1-36.

[3] H. Ghasemi and C. Cañizares, "Confidence intervals estimation in the identification of electromechanical modes from ambient noise," IEEE Transactionson Power Systems, vol. 23, no. 2, pp. 641 -648, May 2008.

[4] H. Ghasemi, C. Cañizares, and A. Moshref, "Oscillatory stability limit prediction using stochastic subspace identification," IEEE Transactions on PowerSystems, vol. 21, no. 2, pp. 736 - 745, May 2006.

[5] D. Trudnowski, "Estimating Electromechanical Mode Shape From Synchrophasor Measurements," IEEE Transactions on Power Systems, vol. 23, no. 3, pp. 1188-1195, Aug. 2008.

[6] S. Haykin, Ed., Nonlinear Methods of Spectral Analysis, (2nd ed. Berlin: Springer-Verlag, 1983).

[7] J. Proakis and D. Manolakis, Digital Signal Processing Principles, Algorithms, and Applications,( 4th ed. Upper Saddle River, New Jersey: PrenticeHall, 2007).

[8] P. Stoica and R. Moses, Spectral Analysis of Signals. (Upper Saddle River, New Jersey: Pearson Prentice Hall, 2005).

[9] S. M. Kay, Modern Spectral Estimation: Theory and Application, (Englewwod Cliffs, NJ: Prentice-Hall, 1988).

[10] D. Trudnowski, J. Pierre, N. Zhou, J. Hauer, and M. Parashar, "Performance of Three Mode-Meter Block-Processing Algorithms for Automated Dynamic Stability Assessment," IEEE Transactions on Power Systems, vol. 23, no. 2, pp. 680-690, May 2008.

[11] J. Hauer, N. Bhatt, K. Shah, and S. Kolluri, "Performance of "wams east" in providing dynamic information for the north east blackout of august 14, 2003," in IEEE Power Engineering Society General Meeting, 2004, June 2004, pp. 1685-1690 Vol.2.

[12] L. Vanfretti, T. M. L. Assis, J. H. Chow, L. Dosiek, J. W. Pierre, D. Trudnowski, and Y. Liu, "Data Analysis of the 2/26/08 Florida Blackout ," NASPI Work Group Meeting, Sacramento, CA, June 4, 2009, availableonline:http://www.naspi.org/meetings/workgroup/workgroup.stm.

[13] G. Ledwich and E. Palmer, "Modal estimates from normal operation of power systems," in Power Engineering Society Winter Meeting, 2000. IEEE, vol. 2, 2000, pp. 1527-1531 vol.2.

[14] D. Vowles, M. Gibbard, D. Geddey, and D. Bones, "Benchmark testing methodology for continuous modal-estimation algorithms," in Power \& Energy Society General Meeting, 2009. PES '09. IEEE, July 2009, pp. 1-8.

[15] Signal Processing ToolboxTM6 User's Guide, The MathWorks, Inc., September 2009.

[16] D. Trudnowski and J.W. Pierre, in Inter-area Oscillations in Power Systems: A Nonlinear and Nonstationary Perspective, ser. Power Electronics and Power Systems, A. R. Messina, Ed. Springer, 2009, ch. Signal Processing Methods for Estimating Small-Signal Dynamic Propertiesfrom Measured Responses, pp. 1-36.

[17] Li Qi, LeweiQian, Stephen Woodruff, and David Cartes, "Prony Analysis for Power System Transient Harmonics", EURASIP Journal on Advances in signal Processing, Vol. 2007, doi:10.1155/2007/48406.

[18] A. Hauer, D. Trudnowski, and J. DeSteese, "A Perspective on WAMS Analysis Tools for Tracking of Oscillatory Dynamics," IEEE Power Engineering Society General Meeting, 2007. Pp. 1-10, June 2007.

[19] Dennis Ward Ricker (2003). Echo Signal Processing. Springer. ISBN 1-4020-7395-X Last Update February 20,2013

[20] M. B. Priestley, Spectral Analysis and Time Series. (London, U.K.:Academic, 1981).

[21] S. Chandra and Lin Wen. Experimental comparison between stationary and non-stationary formulations of linear prediction applied to voiced speech analysis. Acoustics, Speech, and Signal processing [see also IEEE Transactions on Signal Processing], IEEE Transactions on, 22(6):403-415, 1974. 